\title{
TEST SPACES FOR METRIC SPACES ${ }^{1}$
}

\section{BYRON H. MCCANDLESS}

Introduction. Let $n$ be a positive integer, and let $Y^{n}$ be a topological space with the following property: a topological space $X$ has dimension $\leqq n$ if, and only if, given a closed subset $C$ of $X$, any continuous mapping $f: C \rightarrow Y^{n}$ can be extended over $X$. Then we call $Y^{n}$ a test space for dimension $n$. In a previous paper [10], we characterized test spaces for dimension $n$ under the assumption that both $X$ and $Y^{n}$ were separable metric. In addition, we required $Y^{n}$ to be compact, along with some other side conditions. In the present paper, we are able to improve the characterization in two ways. First, we remove the separability requirement from both $X$ and $Y^{n}$, and secondly, the compactness requirement on $Y^{n}$ is removed. The resulting space $Y^{n}$ is called a test space for metric spaces of dimension $n$, or simply a test space for metric spaces. The main reason that we required $Y^{n}$ to be compact in [10] was that compactness was needed to apply S. T. Hu's theorem on the homotopy type of spaces [8]. In the present paper we employ a theorem of J. H. C. Whitehead [11, Theorem 3, p. 216] which allows us to lift this restriction.

Preliminaries. In this paper, $\operatorname{dim} X$ will always mean the dimension of $X$ defined in terms of finite open coverings. Insofar as metric spaces are concerned, this definition is equivalent to the one employed by Kodama in [9], so that his results are applicable here. To avoid trivialities, all spaces considered will be nonempty. Whenever homology groups are mentioned, it is assumed that the singular homology groups are meant.

Let $Y$ be an $n$-dimensional metric space which is $\mathrm{LC}^{n}$, that is, locally connected in all dimensions $\leqq n$ in the sense of homotopy. Then a theorem of Dugundji $[4$, p. 366] shows that $Y$ is dominated by a CW polytope $P$ of dimension $\leqq n$. Note that $P$ is a CW complex in the sense of J. H. C Whitehead [11]. Dugundji also shows that the homology groups of $Y$ are direct summands of the corresponding homology groups of $P$. Now it is known that the singular homology groups of $P$ are isomorphic to the corresponding combinatorial homology groups of $P$ (see $\left[5\right.$, p. 566]) and therefore $H_{i}(P)=\mathrm{C}$ for $i>\operatorname{dim} P$. It follows that $H_{i}(Y)=0$ for $i>\operatorname{dim} Y=n$. Now suppose that $Y$ has a nonzero $n$-dimensional homology group $H_{n}(Y)$. Since

Received by the editors September 3, 1957 and, in revised form, April 7, 1958.

1 Research supported by the Research Council of Rutgers University. 
$H_{n}(Y)$ is a direct summand of $H_{n}(P)$, we have $H_{n}(P) \neq 0$, and therefore $\operatorname{dim} P=n$. Thus we conclude that an $n$-dimensional $\mathrm{LC}^{n}$ metric space $Y$ with $H_{n}(Y) \neq 0$ is dominated by an $n$-dimensional CW polytope $P$.

Note, moreover, that if the space $Y$ is connected, then $P$ is also connected. For $P$ is the nerve $N(V)$ of a certain covering $\{V\}$ of $Y$ by open sets, and is easily shown to be connected as follows: Let $a, b$ be any two vertices of $P$, and let $V_{a}, V_{b}$ be the corresponding open sets of $\{V\}$. Select points $p \in V_{a}, q \in V_{b}$. Then there exists [6] a simple chain consisting of a finite number of elements of $\{V\}$ whose first member is $V_{a}$ and last member is $V_{b}$. This provides a finite sequence of one-simplexes connecting $a$ with $b$, and shows that $P$ is connected.

In case $Y$ is separable, then the coverings can be chosen so that the polytope $P$ is locally finite, and if $Y$ is compact, the coverings can be chosen so that $P$ has a finite number of vertices $[5$, p. 366].

We recall that a topological space $Y$ is aspherical if $\pi_{i}(Y)=0$ for $i>1$.

\section{Lemma 1. A one-dimensional metric space $Y$ is aspherical.}

Proof. This was proved by Curtis and Fort in [2] under the added assumption that $Y$ be separable. To extend their result to the case $Y$ nonseparable, consider any continuous mapping $f: S^{n} \rightarrow Y$, where $n \geqq 2$. Then $f$ has a monotone-light factorization $f=l m$, where $m: S^{n} \rightarrow X$ is monotone, $l: X \rightarrow Y$ is light, and $X$ is the middle space. Now the space $f\left(S^{n}\right)=l(X) \subset Y$ is compact metric, and hence separable. Therefore the proof of Curtis and Fort is valid in this case also.

Given two topological spaces $X$ and $Y$, we write $X \tau Y$ to mean that, given any closed subset $C$ of $X$, any continuous mapping $f: C \rightarrow Y$ has an extension over $X$. As is customary, $\pi_{0}(Y)=0$ will mean that $Y$ is arc-wise connected. Given a positive integer $n$, we consider the implications between the following five properties of a space $Y^{n}$, the first four of which are the same as in [10].

$1^{n} . X \tau Y^{n}$ implies $\operatorname{dim} X \leqq n$.

$2^{n}$. $\operatorname{dim} X \leqq n$ implies $X \tau Y^{n}$.

$3^{n} . \pi_{i}\left(Y^{n}\right)=0, i=0,1, \cdots, n-1$.

$4^{n}$. $Y^{n}$ is $\mathrm{LC}^{n-1}$.

$5^{n} . \pi_{n}\left(Y^{n}\right)$ is infinite cyclic if $n=1$; it is a free abelian group if $n \geqq 2$.

The characterization of test spaces for metric spaces. Let $X$ and $Y^{n}$ be arbitrary metric spaces, with no separability assumed. Note that if $n \geqq 1$ and $Y^{n}$ has property $2^{n}$, then $\pi_{0}\left(Y^{n}\right)=0$. Thus any space $Y^{n}$ which we consider will be arcwise connected. 
Kodama in [9] has proved the following theorem:

THEOREM 1. If $n$ is a positive integer, then a space $Y^{n}$ has property $2^{n}$ if and only if it has properties $3^{n}$ and $4^{n}$.

The following lemma now follows as in [10]:

Lemma 2. Let $n$ be an integer $\geqq 1$. If $Y^{n}$ is $n$-LC and has properties $1^{n}$ and $2^{n}$, then $\pi_{n}\left(Y^{n}\right) \neq 0$.

The next theorem is similar to that in [10], but requires a different proof due to the fact that $Y^{n}$ is not assumed to be compact.

THEOREM 2. Let $n$ be a positive integer and let $Y^{n}$ be an $n$-dimensional $n$-LC metric space. If $n=1$, we also assume that $Y^{1}$ is 1 -simple. If $Y^{n}$ has properties $1^{n}$ and $2^{n}$, it also has property $5^{n}$. Furthermore, $\pi_{n}\left(Y^{n}\right)$ has a finite basis whenever $Y^{n}$ is compact, and an at most countable basis whenever $Y^{n}$ is separable.

Proof. In view of the hypotheses on $Y^{n}$, we have $\pi_{n}\left(Y^{n}\right) \neq 0$ by Lemma 2 . As previously remarked, $Y^{n}$ is dominated by a connected $n$-dimensional CW polytope $P^{n}$, so that the homology groups $H_{i}\left(Y^{n}\right)$ are direct summands of the corresponding groups $H_{i}\left(P^{n}\right)$. If $n=1$, since $Y^{1}$ is 1 -simple, we have $\pi_{1}\left(Y^{1}\right) \approx H_{1}\left(Y^{1}\right)$, and if $n \geqq 2$ the Hurewicz isomorphism theorem implies that $\pi_{n}\left(Y^{n}\right) \approx H_{n}\left(Y^{n}\right)$. Hence, for all $n, \pi_{n}\left(Y^{n}\right) \approx H_{n}\left(Y^{n}\right) \subset H_{n}\left(P^{n}\right)=Z_{n}\left(P^{n}\right)$, where $Z_{n}\left(P^{n}\right)$ is the group of $n$-dimensional cycles of $P^{n}$. Being a subgroup of a free group, $H_{n}\left(Y^{n}\right)$ is free. If $Y^{n}$ is compact, then $P^{n}$ has a finite number of vertices. Therefore the number of cells is finite, so $Z_{n}\left(P^{n}\right)=H_{n}\left(P^{n}\right)$ has a finite number of generators. If $Y^{n}$ is separable, then $P^{n}$, being connected, has at most a countable number of vertices. Thus $P^{n}$ has at most a countable numbers of cells, so that $Z_{n}\left(P^{n}\right)=H_{n}\left(P^{n}\right)$ has an at most countable number of generators. The result now follows if $n \geqq 2$. If $n=1$, Lemma 1 shows that $Y^{1}$ is aspherical. Therefore $Y^{1}$ is of the same homotopy type as a toroid (i.e., the Cartesian product of circles). But any toroid with more than one factor has a nonzero two dimensional homology group, and this would imply that $H_{2}\left(Y^{1}\right) \neq 0$. This, however, is not possible, since $Y^{1}$ is dominated by a onedimensional CW polytope. Therefore $\pi_{1}\left(Y^{1}\right)$ is infinite cyclic, and the proof is complete.

A metric space $Y$ is called an absolute neighborhood retract (metric) if, in every metric space $Z \supset Y$ in which $Y$ is closed, there is a neighborhood $V \supset Y$ of which $Y$ is a retract. According to Kodama $[9$, p. 79], an $n$-dimensional metric space $Y$ is an absolute neighborhood retract (metric) if and only if $Y$ is $\mathrm{LC}^{n}$. Since Borsuk's homo- 
topy extension theorem holds for arbitrary metric spaces, the following lemma is proved exactly as in [10].

Lemma 3. Let $Y$ and $Z$ be absolute neighborhood retracts (metric) with the same homotopy type. Then $Y$ is a test space for metric spaces of dimension $n$ if and only if $Z$ is.

Let $S_{k}^{n}$ be the union of $k n$-spheres $S_{1}^{n}, \cdots, S_{\mathbf{k}}^{n}$ with a single common point, where we allow $k$ to be infinite. If $k$ is infinite, we assume that $S_{\mathbf{k}}^{n}$ is topologized so as to become a CW polytope. Blakers and Massey have shown $\left[1\right.$, p. 200] that the homotopy groups $\pi_{q}\left(S_{\mathbf{k}}^{n}\right)$ are isomorphic to the direct sum of the groups $\pi_{q}\left(S_{\imath}^{n}\right)$ for $q \leqq 2 n-2$. Therefore $\pi_{n}\left(S_{\mathbf{k}}^{n}\right)$ is isomorphic to the direct sum of the groups $\pi_{n}\left(S_{\mathfrak{l}}^{n}\right)$ for $n \geqq 2$.

LeMma 4. $S_{\mathbf{k}}^{n}$ is a test space for metric spaces of dimension $n$.

This easily follows as in [10], since the $n$-sphere tests the dimension of arbitrary metric spaces [3, p. 212].

TheOREM $3 .^{2}$ Let $n$ be an integer $\geqq 1$ and let $Y^{n}$ be an $n$-dimensional $n$-LC metric space. If $Y^{n}$ has properties $3^{n}, 4^{n}$ and $5^{n}$, then $Y^{n}$ also has property $1^{n}$.

Proof. If $n=1$, since $\pi_{1}\left(Y^{1}\right) \approx \pi_{1}\left(\delta_{1}^{1}\right)$, and $Y^{1}, s_{1}^{1}$ are aspherical, it follows that $Y^{1}$ and $\delta_{1}^{\prime}$ have the same homotopy type. Since $Y^{1}$ and $\delta_{1}^{1}$ are absolute neighborhood retracts, Lemma 3 shows that $Y^{1}$ is a test space for metric spaces of dimension one, and consequently has property $1^{1}$.

Now let $n \geqq 2$. By property $5^{n}$, we first select $k$ (finite or infinite) so that $H_{n}\left(\mathcal{S}_{\mathbf{k}}^{n}\right) \approx H_{n}\left(Y^{n}\right)$. As has already been noted, $H_{i}\left(Y^{n}\right)=0$ for $i>n$. Since we also have $H_{i}\left(Y^{n}\right)=0$ for $i<n$ (by property $3^{n}$ ), it follows that $H_{n}\left(Y^{n}\right)$ is the only nonzero homology group of $Y^{n}$. Similarly, $H_{n}\left(S_{k}^{n}\right)$ is the only nonvanishing homology group of $S_{k}^{n}$. Now Eilenberg's classification theorem is applicable in this case, even though $S_{k}^{n}$ is not necessarily locally finite [12 p. 101]. Therefore, since $H^{i}\left(S_{\boldsymbol{k}}^{n}, \pi_{i}\left(Y^{n}\right)\right) \approx H^{i+1}\left(S_{\boldsymbol{k}}^{n}, \pi_{i}\left(Y^{n}\right)\right)=0$ for $i>n$, the homotopy classes of continuous mappings $f: S_{k}^{n} \rightarrow Y^{n}$ are in 1-1 correspondence with the elements of $H^{n}\left(\mathcal{S}_{k}^{n}, \pi_{n}\left(Y^{n}\right)\right) \approx H^{n}\left(\mathcal{S}_{k}^{n}, H_{n}\left(Y^{n}\right)\right)$. Since $H_{n-1}\left(S_{\mathbf{k}}^{n}\right)=0$, we have $H^{n}\left(\mathcal{S}_{\mathbf{k}}^{n}, H_{n}\left(Y^{n}\right)\right) \approx \operatorname{Hom}\left[H_{n}\left(S_{\mathbf{k}}^{n}\right), H_{n}\left(Y^{n}\right)\right][12$, p. 102]. Therefore the isomorphism $H_{n}\left(\mathcal{S}_{\mathbf{k}}^{n}\right) \approx H_{n}\left(Y^{n}\right)$ can be realized by a continuous mapping $f: S_{\boldsymbol{k}}^{n} \rightarrow Y^{n}$. Whitehead's theorem [11, p. 216]

2 The author is indebted to the referee for suggesting how to remove the separability condition from $Y^{n}$ in Theorem 3. 
now shows that $S_{\mathbf{k}}^{n}$ and $Y^{n}$ have the same homotopy type. Finally, we note that since $Y^{n}$ is $n$-LC and has property $4^{n}, Y^{n}$ is $\mathrm{LC}^{n}$. Hence Kodama's result shows that $Y^{n}$ is an absolute neighborhood retract (metric). By virtue of Lemmas 3 and $4, Y^{n}$ is a test space for metric spaces of dimension $n$ and therefore has property $1^{n}$. The proof is now complete.

Combining Theorems 1, 2, and 3, we finally obtain

THEOREM 4. Let $n$ be an integer $\geqq 1$ and let $Y^{n}$ be an $n$-dimensional, $n$-LC metric space. If $n=1$, we also assume that $Y^{1}$ is 1 -simple. Then $Y^{n}$ is a test space for metric spaces of dimension $n$ if and only if (i) $\pi_{i}\left(Y^{n}\right)=0, i=0,1, \cdots, n-1$. (ii) $Y^{n}$ is $\mathrm{LC}^{n-1}$. (iii) $\pi_{n}\left(Y^{n}\right)$ is infinite cyclic if $n=1$; it is a free abelian group if $n \geqq 2$.

\section{BIBLIOGRAPHY}

1. A. L. Blakers and W. S. Massey, The homotopy groups of a triad I, Ann. of Math. vol. 53 (1951) pp. 161-205.

2. M. L. Curtis and M. K. Fort, Jr., Homotopy groups of one-dimensional spaces, Proc. Amer. Math. Soc. vol. 8 (1957) p. 578.

3. C. H. Dowker, Mapping theorems for noncompact spaces, Amer. J. Math. vol. 69 (1947) pp. 200-241.

5. J. Dugundji, An extension of Tietze's theorem, Pacific J. Math. vol. 1 (1951) pp. 353-367.

6. S. Eilenberg, Continuous mappings of infinite polyhedra, Ann. of Math. vol. 42 (1941) pp. 459-468.

7. D. W. Hall and G. L. Spencer, Elementary topology, Wiley, 1955, p. 81 .

8. S. T. Hu, Mappings of a normal space into an absolute neighborhood retract, Trans. Amer. Math. Soc. vol. 64 (1948) pp. 336-358.

9. Y. Kodama, On LC $C^{n}$ metric spaces, Proc. Japan Acad. vol. 33 (1957) pp. 79-83.

10. B. H. McCandless, Test spaces for dimension n, Proc. Amer. Math. Soc. vol. 7 (1956) pp. 1126-1130.

11. J. H. C. Whitehead, Combinatorial homotopy I, Bull. Amer. Math. Soc. vol. 55 (1949) pp. 213-245.

12. G. W. Whitehead, Homotopy theory, mimeographed notes compiled by Robert J. Aumann.

RUTGERS UNIVERSITY 